\title{
A NOTE ON MEAN EQUICONTINUITY
}

\author{
JIAHAO QIU AND JIANJIE ZHAO
}

\begin{abstract}
In this note, it is shown that several results concerning mean equicontinuity proved before for minimal systems are actually held for general topological dynamical systems. Particularly, it turns out that a dynamical system is mean equicontinuous if and only if it is equicontinuous in the mean if and only if it is Banach (or Weyl) mean equicontinuous if and only if its regionally proximal relation is equal to the Banach proximal relation.

Meanwhile, a relation is introduced such that the smallest closed invariant equivalence relation containing this relation induces the maximal mean equicontinuous factor for any system.
\end{abstract}

\section{INTRODUCTION}

Throughout this paper, a topological dynamical system is a pair $(X, T)$, where $X$ is a non-empty compact metric space with a metric $d$ and $T$ is a continuous map from $X$ to itself.

We all know that equicontinuous systems have simple dynamical behaviors. By the well known Halmos-von Neumann theorem, a transitive equicontinuous system is conjugate to a minimal rotation on a compact abelian metric group, and $(X, T, \mu)$ has discrete spectrum, where $\mu$ is the unique Haar measure on $X$. In this note, we discuss the systems with equicontinuity in the mean sense.

Recall that a dynamical system $(X, T)$ is called mean equicontinuous if for every $\varepsilon>0$, there exists a $\delta>0$ such that whenever $x, y \in X$ with $d(x, y)<\delta$, we have

$$
\limsup _{n \rightarrow \infty} \bar{d}_{n}(x, y)<\varepsilon, \text { where } \bar{d}_{n}(x, y)=\frac{1}{n} \sum_{i=0}^{n-1} d\left(T^{i} x, T^{i} y\right) .
$$

A notion called stable in the mean in the sense of Lyapunov or simply mean-L-stable is introduced by Fomin [4]. We call a dynamical system $(X, T)$ mean-L-stable if for every $\varepsilon>0$, there is a $\delta>0$ such that $d(x, y)<\delta$ implies $d\left(T^{n} x, T^{n} y\right)<\varepsilon$ for all $n \in \mathbb{Z}_{+}$except a set of upper density less than $\varepsilon$. Oxtoby [14], Auslander [1] and Scarpellini [15] also studied mean-L-stable systems. It is easy to see that a dynamical system is mean-L-stable if and only if it is mean equicontinuous. Answering an open question in [15], it was proved by $\mathrm{Li}, \mathrm{Tu}$ and $\mathrm{Ye}$ in [12] that a minimal mean equicontinuous system has discrete spectrum. We refer to $[6,7,8,9,10,13]$ for further study on mean equicontinuity and related subjects.

In the study of a dynamical system with bounded complexity (defined by using the mean metrics), recently Huang, Li, Thouvenot, Xu and Ye [10] introduced a notion called

Date: November 16, 2018.

2010 Mathematics Subject Classification. 54H20, 37A25.

Key words and phrases. Mean equicontinuity, equicontinuity in the mean. 
equicontinuity in the mean. We say that a dynamical system $(X, T)$ is equicontinuous in the mean if for every $\varepsilon>0$, there exists a $\delta>0$ such that $\frac{1}{n} \sum_{i=0}^{n-1} d\left(T^{i} x, T^{i} y\right)<\varepsilon$ for all $n \in \mathbb{Z}_{+}$and all $x, y \in X$ with $d(x, y)<\delta$. It was proved in [10] that for a minimal system the notions of mean equicontinuity and equicontinuity in the mean are equivalent. In this note we will show that a dynamical system is mean equicontinuous if and only if it is equicontinuous in the mean (Theorem 3.8).

In [12] the notion of Banach (or Weyl) mean equicontinuity was introduced, and the authors asked if for a minimal system Banach mean equicontinuity is equal to mean equicontinuity. This question was answered positively in [2]. In this note we will show that in fact for any system the two notions are equivalent (Theorem 5.1). Moreover, in [12] the authors showed that if $(X, T)$ is mean equicontinuous, then its regionally proximal relation is equal to the Banach proximal relation. In this note we will prove that the converse statement is also valid (Theorem 4.3).

Moreover, we define a notion called regionally proximal relation in the mean and we show that the mean equicontinuous structure relation is the smallest closed invariant equivalence relation that contains regionally proximal relation in the mean (Theorem 6.6).

The note is organized as follows. In Section 2, the basic notions used in the note are introduced. In Section 3, among other things we show that mean equicontinuity is equal to equicontinuity in the mean. In Section 4 , we prove that if the regionally proximal relation is equal to Banach proximal relation then the system is mean equicontinuous. In Section 5, we prove the equivalence of mean equicontinuity and Weyl mean equicontinuity. In the final section, we discuss the question which relation induces the maximal mean equicontinuous factor.

\section{PRELIMINARIES}

In this section we recall some notions and aspects of the theory of topological dynamical systems.

2.1. Subsets of non-negative integers. Let $\mathbb{Z}_{+}(\mathbb{N}, \mathbb{Z}$, respectively) be the set of all nonnegative integers (positive integers, integers, respectively).

Let $F$ be a subset of $\mathbb{Z}_{+}$(NN, $\mathbb{Z}$, respectively). Denote by $\#(F)$ the number of elements of $F$.

We say that $F$ has density $D(F)$ if the lower density of $F(\underline{D}(F))$ is equal to the upper density of $F(\bar{D}(F))$, that is, $D(F)=\bar{D}(F)=\underline{D}(F)$, where

$$
\underline{D}(F)=\liminf _{n \rightarrow \infty} \frac{\#(F \cap[0, n-1])}{n}
$$

and

$$
\bar{D}(F)=\limsup _{n \rightarrow \infty} \frac{\#(F \cap[0, n-1])}{n} .
$$

Similarly, we say that $F$ has Banach density if the lower Banach density of $F\left(B D_{*}(F)\right)$ is equal to the upper Banach density of $F\left(B D^{*}(F)\right)$, that is, $B D(F)=B D_{*}(F)=B D^{*}(F)$, where,

$$
B D_{*}(F)=\liminf _{N-M \rightarrow \infty} \frac{\#(F \cap[M, N])}{N-M+1}
$$


and

$$
B D^{*}(F)=\limsup _{N-M \rightarrow \infty} \frac{\#(F \cap[M, N])}{N-M+1} .
$$

2.2. Compact metric spaces. Denote by $(X, d)$ a compact metric space. For $x \in X$ and $\varepsilon>0$, denote $B(x, \varepsilon)=\{y \in X: d(x, y)<\varepsilon\}$. We denote by $\operatorname{diam}(X)$ the diameter of $X$ given by $\operatorname{diam}(X)=\sup _{x, y \in X} d(x, y)$, the product space $X \times X=\{(x, y): x, y \in X\}$ and the diagonal $\Delta_{X}=\{(x, x): x \in X\}$.

Let $C(X)$ be the set of continuous complex value functions on $X$ with the supremum norm $\|f\|=\sup _{x \in X}|f(x)|$. We denote by $C(X)^{*}$ the dual space of $C(X)$.

2.3. Topological dynamics. Let $(X, T)$ be a dynamical system. A non-empty closed invariant subset $Y \subset X$ (i.e., $T Y \subset Y)$ defines naturally a subsystem $(Y, T)$ of $(X, T)$. A system $(X, T)$ is called minimal if it contains no proper subsystem. Each point belonging to some minimal subsystem of $(X, T)$ is called a minimal point. The orbit of a point $x \in X$ is the set $\operatorname{Orb}(x, T)=\left\{x, T x, T^{2} x, \ldots,\right\}$. The set of limit points of the orbit $\operatorname{Orb}(x, T)$ is called the $\omega$-limit set of $x$, and is denoted by $\omega(x, T)$. For $x \in X$ and $U, V \subset X$, put $N(x, U)=\left\{n \in \mathbb{Z}_{+}: T^{n} x \in U\right\}$ and $N(U, V)=\left\{n \in \mathbb{Z}_{+}: U \cap T^{-n} V \neq \emptyset\right\}$. Recall that a dynamical system $(X, T)$ is called topologically transitive (or just transitive) if for every two non-empty open subsets $U, V$ of $X$ the set $N(U, V)$ is infinite. Any point with dense orbit is called a transitive point. Denote the set of all transitive points by $\operatorname{Trans}(X, T)$. It is well known that for a transitive system, $\operatorname{Trans}(X, T)$ is a dense $G_{\delta}$ subset of $X$.

For two dynamical systems $(X, T)$ and $(Y, S)$. Let $\pi: X \rightarrow Y$ be a continuous map. If $\pi$ is surjective with $\pi \circ T=S \circ \pi$, then we say that $\pi$ is a factor map, the system $(Y, T)$ is a factor of $(X, T)$ or $(X, T)$ is an extension of $(Y, T)$. If $\pi$ is a homeomorphism, then we say that $\pi$ is a conjugacy and that the dynamical systems $(X, T)$ and $(Y, S)$ are conjugate. By the Halmos and von Neumann theorem (see [16, Theorem 5.18]), a minimal system is equicontinuous if and only if it is conjugate to a minimal rotation on a compact abelian metric group.

A pair $(x, y) \in X \times X$ is said to be proximal if for any $\varepsilon>0$, there exists a positive integer $n$ such that $d\left(T^{n} x, T^{n} y\right)<\varepsilon$. Let $P(X, T)$ denote the collection of all proximal pairs in $(X, T)$. If any pair of two points in $X$ is proximal, then we say that the dynamical system $(X, T)$ is proximal.

A pair $(x, y) \in X \times X$ is said to be Banach proximal if for any $\varepsilon>0, d\left(T^{n} x, T^{n} y\right)<\varepsilon$ for all $n \in \mathbb{Z}_{+}$except a set of zero Banach density. Let $B P(X, T)$ denote the collection of all Banach proximal pairs in $(X, T)$. See [11] for a detailed study on Banach proximality.

A pair $(x, y)$ is called regionally proximal if for every $\varepsilon>0$, there exist two points $x^{\prime}, y^{\prime} \in X$ with $d\left(x, x^{\prime}\right)<\varepsilon$ and $d\left(y, y^{\prime}\right)<\varepsilon$, and a positive integer $n$ such that $d\left(T^{n} x^{\prime}, T^{n} y^{\prime}\right)<$ $\varepsilon$. Let $Q(X, T)$ be the set of all regionally proximal pairs in $(X, T)$. Clearly, $Q(X, T) \supset$ $P(X, T) \supset B P(X, T)$.

A factor map $\pi:(X, T) \rightarrow(Y, S)$ is called proximal (Banach proximal, respectively) if whenever $\pi(x)=\pi(y)$ the pair $(x, y)$ is proximal (Banach proximal, respectively ).

The factor $\pi:(X, T) \rightarrow(Y, S)$ is the maximal equicontinuous factor if the system $(Y, T)$ is equicontinuous and for any other factor map $\phi:(X, T) \rightarrow(Z, U)$, where $(Z, U)$ is equicontinuous, there exists a factor map $\psi:(Y, S) \rightarrow(Z, U)$ such that $\phi=\psi \circ \pi$. It is thus unique up to conjugacy and therefore referred to as the maximal equicontinuous factor. Let $\pi:(X, T) \rightarrow(Y, S)$ be the factor map to the maximal equicontinuous factor. 
The equivalence relation $R_{\pi}=\{(x, y) \in X \times X: \pi(x)=\pi(y)\}$ is called the equicontinuous structure relation. It is shown in [3] that the equicontinuous structure relation is the smallest closed invariant equivalence relation containing the regionally proximal relation.

2.4. Invariant measures. For a dynamical system $(X, T)$, we denote by $M(X, T)$ the set of $T$-invariant regular Borel probability measures on $X$. It is well known that $M(X, T)$ is always nonempty. We say that $(X, T)$ is uniquely ergodic if $M(X, T)$ consists a single measure. We regard $M(X)$ as a closed convex subset of $C(X)^{*}$, equipped with the weak* topology. Then $M(X)$ is a compact metric space. An invariant measure is ergodic if and only if it is an extreme point of $M(X, T)$.

Let $\mu \in M(X, T)$. We define the support of $\mu$ by $\operatorname{supp}(\mu)=\{x \in X: \mu(U)>0$ for any neighborhood $U$ of $x\}$. The support of a dynamical system $(X, T)$, denoted by $\operatorname{supp}(X, T)$, is the smallest closed subset $C$ of $X$ such that $\mu(C)=1$ for all $\mu \in M(X, T)$.

The action of $T$ on $X$ induces an action on $M(X)$ in the following way: for $\mu \in M(X)$ we define $T \mu$ by

$$
\int_{X} f(x) \mathrm{d} T \mu(x)=\int_{X} f(T x) \mathrm{d} \mu(x), \quad \forall f \in C(X) .
$$

Hence $(M(X), T)$ is also a topological dynamical system.

Fix a measure space $(X, \mathscr{B}, \mu)$. If $f$ and $g$ are functions on $X$, we denote by $f \otimes g$ the function on $X \times X$ given by $f \otimes g\left(x, x^{\prime}\right)=f(x) g\left(x^{\prime}\right)$ and by $L^{\infty}(X) \otimes L^{\infty}(X)$ we denote the algebra of functions on $X \times X$ that are finite sums of functions $f \otimes g$ with $f, g \in$ $L^{\infty}(X, \mathscr{B}, \mu)$. We denote by $\mu_{\Delta}$ the diagonal measure on $X \times X$ given by $\int f\left(x, x^{\prime}\right) \mathrm{d} \mu_{\Delta}\left(x, x^{\prime}\right)=$ $\int f(x, x) \mathrm{d} \mu(x)$. We notice that $\mu_{\Delta}(A \times B)=\mu(A \cap B)$ for any $A, B \in \mathscr{B}$.

For a dynamical system $(X, T), f \in C(X)$ and $n \in \mathbb{N}$, let $f_{n}(x)=\frac{1}{n} \sum_{i=0}^{n-1} f\left(T^{i} x\right)$. The following theorem is well known.

Theorem 2.1. [14] Let $(X, T)$ be a dynamical system. Then the following conditions are equivalent:

(1) $(X, T)$ is uniquely ergodic;

(2) for each $f \in C(X),\left\{f_{n}\right\}_{n=1}^{\infty}$ converges uniformly on $X$ to a constant;

(3) for each $f \in C(X)$, there is a subsequence $\left\{f_{n_{k}}\right\}_{k=1}^{\infty}$ which converges pointwise on $X$ to a constant.

(4) $(X, T)$ contains only one minimal set, and for each $f \in C(X),\left\{f_{n}\right\}_{n=1}^{\infty}$ converges uniformly on $X$.

\section{MEAN EQUICONTINUITY AND EQUICONTINUITY IN THE MEAN}

In this section we will show that mean equicontinuity is equal to equicontinuity in the mean. Moreover, we will discuss what kinds of minimal sets can appear in a transitive mean equicontinuous system.

3.1. Mean equicontinuity and equicontinuity in the mean. We start with the following characterizations of equicontinuous in the mean systems. To do this, we need a simple lemma.

Lemma 3.1. [12, Lemma 3.2] Let $(X, T)$ and $(Y, S)$ be two dynamical systems. Then $(X \times Y, T \times S)$ is mean equicontinuous if and only if both $(X, T)$ and $(Y, S)$ are mean equicontinuous. 
Theorem 3.2. Let $(X, T)$ be a dynamical system. Then the following conditions are equivalent:

(1) $(X, T)$ is equicontinuous in the mean;

(2) for each $f \in C(X \times X)$, the sequence $\left\{\frac{1}{n} \sum_{i=0}^{n-1} f \circ\left(T^{i} \times T^{i}\right)\right\}_{n=1}^{\infty}$ is uniformly equicontinuous;

(3) for each $f \in C(X \times X)$, the sequence $\left\{\frac{1}{n} \sum_{i=0}^{n-1} f \circ\left(T^{i} \times T^{i}\right)\right\}_{n=1}^{\infty}$ is uniformly convergent to a $T \times T$-invariant continuous function $f^{*} \in C(X \times X)$.

Proof. We only present the proof (1) implies (2) and the rest is similar to the proof of [12, Theorem 3.3].

To make the idea of the proof clearer, when proving $(1) \Rightarrow(2)$, we assume $f \in C(X)$ instead of $f \in C(X \times X)$, because if $(X, T)$ is equicontinuous in the mean if and only if so is $(X \times X, T \times T)$.

$(1) \Rightarrow(2)$ Fix $f \in C(X)$ and $\varepsilon>0$. By continuity of $f$, there exists $\eta \in\left(0, \frac{\varepsilon}{2\|f\|}\right)$ such that if $x, y \in X$ with $d(x, y)<\eta$ then $|f(x)-f(y)|<\frac{\varepsilon}{2}$. As $(X, T)$ is equicontinuous in the mean, there is $\delta \in(0, \eta)$ such that if $x, y \in X$ with $d(x, y)<\delta$, one has

$$
\frac{1}{n} \sum_{i=0}^{n-1} d\left(T^{i} x, T^{i} y\right)<\eta^{2}, n=1,2, \ldots
$$

For every $n \in \mathbb{N}$ and $x, y \in X$ with $d(x, y)<\delta$, let

$$
I_{n}(x, y)=\left\{i \in[0, n-1]: d\left(T^{i} x, T^{i} y\right) \geq \eta\right\} .
$$

Then $\#\left(I_{n}(x, y)\right) \leq \eta n$. So for every $n \in \mathbb{N}$ and $x, y \in X$ with $d(x, y)<\delta$, we have

$$
\begin{aligned}
\left|\frac{1}{n} \sum_{i=0}^{n-1} f\left(T^{i} x\right)-\frac{1}{n} \sum_{i=0}^{n-1} f\left(T^{i} y\right)\right| & \leq \frac{1}{n} \sum_{i=0}^{n-1}\left|f\left(T^{i} x\right)-f\left(T^{i} y\right)\right| \\
& \leq \frac{1}{n}\left(\sum_{i \in I_{n}(x, y)} 2\|f\|+\sum_{i \in[0, n-1] \backslash I_{n}(x, y)}\left|f\left(T^{i} x\right)-f\left(T^{i} y\right)\right|\right) \\
& \leq 2 \eta\|f\|+\frac{\varepsilon}{2}<\varepsilon .
\end{aligned}
$$

This shows that $\left\{\frac{1}{n} \sum_{i=0}^{n-1} f \circ T^{i}\right\}_{n=1}^{\infty}$ is uniformly equicontinuous.

Before proving the main result of this section we give a proof of a result in [14] which is outlined there. We need the following lemmas.

Lemma 3.3. [10] Let $(X, T)$ be a minimal dynamical system. Then $(X, T)$ is mean equicontinuous if and only if it is equicontinuous in the mean.

Lemma 3.4. [12, Theorem 3.5] Let $(X, T)$ be a dynamical system. If $(X, T)$ is mean equicontinuous, then $Q(X, T)=P(X, T)=B P(X, T)$ and it is a closed invariant equivalence relation.

Lemma 3.5. [11] Let $(X, T)$ be a dynamical system. Then the support of $(X, T)$ is the smallest closed subset $K$ of $X$ such that for every $x \in X$ and every open neighborhood $U$ of $K, N(x, U)$ has Banach density one.

Now we are ready to show 
Theorem 3.6. Let $(X, T)$ be a dynamical system. If $(X, T)$ is mean equicontinuous, then for every $x \in X,(\overline{O r b(x, T)}, T)$ is uniquely ergodic. In particular, if $(X, T)$ is also transitive, then $(X, T)$ is uniquely ergodic.

Proof. Without loss of generality, we can assume that $X=\overline{\operatorname{Orb}(x, T)}$.

If $M_{1}, M_{2}$ are two minimal sets in $(X, T)$. By Auslanser-Ellis theorem, there exist $y_{1} \in$ $M_{1}$ and $y_{2} \in M_{2}$ such that $\left(x, y_{1}\right)$ and $\left(x, y_{2}\right)$ are both proximal. For a given $\varepsilon>0$, set

$$
A_{1}=\left\{n \in \mathbb{Z}_{+}: d\left(T^{n} x, T^{n} y_{1}\right)<\varepsilon / 2\right\} \text { and } A_{2}=\left\{n \in \mathbb{Z}_{+}: d\left(T^{n} x, T^{n} y_{2}\right)<\varepsilon / 2\right\} .
$$

By Lemma 3.4, $A_{1} \cap A_{2} \neq \emptyset$ which implies that $\left(y_{1}, y_{2}\right)$ is proximal. As $y_{1}$ and $y_{2}$ are minimal points, then their orbit closures are equal which deduces that $M_{1}=M_{2}$. So there is only one minimal set in $(X, T)$, denoted by $M$.

It is clear that $(M, T)$ is also mean equicontinuous. By Lemma 3.3, $(M, T)$ is equicontinuous in the mean. Then $(M, T)$ is uniquely ergodic by Theorem 2.1 and Theorem 3.2. For every $z \in X$, by Auslanser-Ellis theorem again, there exists a point $y \in M$ such that $(z, y)$ is proximal. By Lemma 3.4, $(z, y)$ is a Banach proximal piont. So for every open neighborhood $U$ of $M$ and any $z \in X, N(z, U)$ has Banach density one. Then by Lemma 3.5 we have $\operatorname{supp}(X, T) \subset M$. As $(M, T)$ is uniquely ergodic, so is $(X, T)$.

Now we begin to prove the main result of this section. We need the following lemma.

Lemma 3.7. Let $(X, T)$ be mean equicontinuous system and $v$ be an ergodic measure on $X$, then every point of supp $(v)$ is minimal.

Proof. $(\operatorname{supp}(v), T)$ is a transitive system since $v$ is an ergodic measure on $X$. By Theorem 3.6, $(\operatorname{supp}(v), T)$ is uniquely ergodic, and so, it is minimal.

Now we are ready to show the main result. Note that our method is different from the proof for the minimal case.

Theorem 3.8. $(X, T)$ is mean equicontinuous if and only if equicontinuous in the mean.

Proof. If $(X, T)$ is equicontinuous in the mean, it is clear that $(X, T)$ is mean equicontinuous.

Now assume that $(X, T)$ is mean equicontinuous. If $(X, T)$ is not equicontinuous in the mean, then there are $x_{k}, y_{k}, z \in X, n_{k} \in \mathbb{Z}_{+}, k=1,2, \cdots$ and $\varepsilon_{0}>0$ such that $\lim _{k \rightarrow \infty} x_{k}=$ $z=\lim _{k \rightarrow \infty} y_{k}$ and for every $k \in \mathbb{Z}_{+}$

$$
\frac{1}{n_{k}} \sum_{i=0}^{n_{k}-1} d\left(T^{i} x_{k}, T^{i} y_{k}\right) \geq \varepsilon_{0} \text {. }
$$

Let $\mu_{k}=\frac{1}{n_{k}} \sum_{i=0}^{n_{k}-1} \delta_{\left(T^{i} x_{k}, T^{i} y_{k}\right)}$, then $\mu_{k} \in M(X \times X)$, we may assume $\mu_{k} \rightarrow \mu$ (otherwise we may consider the subsequence), where $\mu \in M(X \times X, T \times T)$.

We claim that $\mu\left(\operatorname{supp}(\mu) \backslash \Delta_{X}\right)>0$. Actually, $d(\cdot, \cdot)$ is a continuous function on $X \times X$, then we have

and

$$
\int_{X \times X} d(x, y) \mathrm{d} \mu_{k} \longrightarrow \int_{X \times X} d(x, y) \mathrm{d} \mu
$$

$$
\int_{X \times X} d(x, y) \mathrm{d} \mu_{k}=\frac{1}{n_{k}} \sum_{i=0}^{n_{k}-1} d\left(T^{i} x_{k}, T^{i} y_{k}\right) \geq \varepsilon_{0}
$$


which implies

$$
\mu\left(\operatorname{supp}(\mu) \backslash \Delta_{X}\right)>0 .
$$

By the ergodic decomposition, we have $v\left(\operatorname{supp}(\mu) \backslash \Delta_{X}\right)>0$ for some ergodic measure on $X \times X$. Thus, there exists a minimal point in $\operatorname{supp}(\mu) \backslash \Delta_{X}$, since $\operatorname{supp}(v)$ is a minimal set by Lemma 3.7. Denote this minimal point by $(u, v)$.

For $l \in \mathbb{N}$, let $B_{l}=\left\{(x, y) \in X \times X: d((x, y),(u, v))<\frac{1}{l}\right\}$, then

$$
\mu\left(B_{l}\right)>0 \text { and } \mu_{k}\left(B_{l}\right)=\frac{1}{n_{k}} \#\left(\left\{0 \leq i \leq n_{k}:\left(T^{i} x_{k}, T^{i} y_{k}\right) \in B_{l}\right\}\right) .
$$

There are infinitely many $k \in \mathbb{Z}_{+}$with $0 \leq m_{k} \leq n_{k}$ such that $\left(T^{m_{k}} x_{k}, T^{m_{k}} y_{k}\right) \in B_{l}$, since $0<\mu\left(B_{l}\right) \leq \liminf _{k \rightarrow \infty} \mu_{k}\left(B_{l}\right)$ for every $l \in \mathbb{N}$.

Put

$$
\delta=d(\overline{\operatorname{Orb}((z, z), T \times T)}, \overline{\operatorname{Orb}((u, v), T \times T)}) .
$$

Then, $\delta>0$, since $\overline{\operatorname{Orb}((u, v), T \times T)} \cap \Delta_{X}=\emptyset$. As $(X, T)$ is mean equicontinuous, so is $(X \times X, T \times T)$ by Lemma 3.1. Then, for $\frac{1}{4} \delta$, there is $\eta>0$ such that if $d\left((x, y),\left(x^{\prime}, y^{\prime}\right)\right)<$ $\eta$, then

$$
\limsup _{n \rightarrow \infty} \frac{1}{n} \sum_{i=0}^{n-1} d\left((T \times T)^{i}(x, y),(T \times T)^{i}\left(x^{\prime}, y^{\prime}\right)\right)<\frac{\delta}{4} .
$$

We can choose $k \in \mathbb{Z}_{+}$with $d\left(\left(x_{k}, y_{k}\right),(z, z)\right)<\eta$ and $d\left(\left(T^{m_{k}} x_{k}, T^{m_{k}} y_{k}\right),(u, v)\right)<\eta$, then

$$
\limsup _{n \rightarrow \infty} \frac{1}{n} \sum_{i=0}^{n-1} d\left((T \times T)^{i}\left(x_{k}, y_{k}\right),(T \times T)^{i}(z, z)\right)<\frac{\delta}{4}
$$

and

$$
\limsup _{n \rightarrow \infty} \frac{1}{n} \sum_{i=0}^{n-1} d\left((T \times T)^{i}\left(T^{m_{k}} x_{k}, T^{m_{k}} y_{k}\right),(T \times T)^{i}(u, v)\right)<\frac{\delta}{4}
$$

which implies

$$
\limsup _{n \rightarrow \infty} \frac{1}{n} \sum_{i=0}^{n-1} d\left((T \times T)^{i}\left(T^{m_{k}} z, T^{m_{k}} z\right),(T \times T)^{i}(u, v)\right)<\frac{\delta}{2} .
$$

It is a contradiction, thus $(X, T)$ is equicontinuous in the mean.

3.2. Minimal sets in a transitive mean equicontinuous system. In Theorem 3.6 we have shown that a transitive mean equicontinuous system is uniquely ergodic, and thus it contains a unique minimal subset. Here we will discuss what kinds of minimal sets can appear in a transitive mean equicontinuous system.

Theorem 3.9. We have the following observations.

(1) If $(X, T)$ is weakly mixing and mean equicontinuous, then the unique minimal set is a fixed point.

(2) If $(X, T)$ is totally transitive and mean equicontinuous, then the unique minimal set is totally minimal and mean equicontinuous. Moreover, any totally transitive, minimal, mean equicontinuous system can be realized in a totally transitive nonminimal mean equicontinuous system. 
(3) If $(X, T)$ is transitive and mean equicontinuous, then the unique minimal set is mean equicontinuous. Moreover, any minimal, mean equicontinuous system can be realized in a transitive non-minimal mean equicontinuous system.

Proof. (1). If $(X, T)$ is mean equicontinuous and weakly mixing, then $(X \times X, T \times T)$ is transitive, thus it is uniquely ergodic by Theorem 3.6. As we know $\mu \times \mu$ and $\mu_{\Delta}$ are invariant measure on $X \times X$ for any invariant measure $\mu$ on $X$, thus $\mu \times \mu=\mu_{\Delta}$. $\mu_{\Delta}\left(\Delta_{X}\right)=1$ implies $\mu$ must be $\delta_{x}$ for some $x \in X$. Hence $(X, T)$ is also uniquely ergodic and the unique fixed point is $x$.

(2). If $(X, T)$ is mean equicontinuous and totally transitive, it has only one minimal set by Theorem 3.6, denoted by $M$. Then $(M, T)$ is totally minimal. Actually, $\left(X, T^{n}\right)$ is also a transitive mean equicontinuous system for every $n \in \mathbb{N}$, again by Theorem 3.6, there is only one $T^{n}$-invariant measure on $X$ denoted by $\mu_{n}$. Let $\mu$ be the unique invariant measure on $(X, T)$ and it is also invariant on $T^{n}$, hence $\mu=\mu_{n}$ and $M=\operatorname{supp}(\mu)=\operatorname{supp}\left(\mu_{n}\right)$, which implies $\left(M, T^{n}\right)$ is minimal. It is clear that $(X, T)$ is mean equicontinuous.

Now let $\left(X_{1}, T_{1}\right)$ be a totally minimal mean equicontinuous system and $\left(X_{2}, T_{2}\right)$ be a weakly mixing system such that the uniquely ergodic measure is supported on a fixed point $p$. Then, $\left(X_{2}, T_{2}\right)$ is mean equicontinuous and $\left(X_{1} \times X_{2}, T_{1} \times T_{2}\right)$ is the system we want.

(3). The first statement follows again by Theorem 3.6. Let $\left(X_{1}, T_{1}\right)$ be a minimal mean equicontinuous system and $\left(X_{2}, T_{2}\right)$ be a weakly mixing system such that the uniquely ergodic measure is supported on a fixed point $p$. Then, $\left(X_{2}, T_{2}\right)$ is mean equicontinuous and $\left(X_{1} \times X_{2}, T_{1} \times T_{2}\right)$ is the system we want.

\section{REGIONALLY PROXIMAL AND BANACH PROXIMAL RELATIONS}

Lemma 3.4 shows that for a mean equicontinuous system $(X, T)$, we have $B P(X, T)=$ $P(X, T)=Q(X, T)$. We will show the converse is also valid, i.e. for a dynamical system $(X, T)$, if $B P(X, T)=P(X, T)=Q(X, T)$ then it is equicontinuous in the mean. In fact we will prove more by providing a series of equivalent statements, see Theorem 4.3 for details.

We start with some preparations. The following lemma is just a simple observation.

Lemma 4.1. Let $(X, T)$ be a dynamical system, if $(x, y) \in B P(X, T)$, then for any neighborhood $U$ of $\Delta_{X}$, we have $B D(N((x, y), U))=1$.

Lemma 4.2. Let $(X, T)$ be a dynamical system. If there exists $\mu \in M(X \times X, T \times T)$ such that $\mu(B P(X, T))=1$, then $\mu\left(\Delta_{X}\right)=1$.

Proof. Assume that $\mu\left(\Delta_{X}\right)<1$, i.e. $\mu\left(B P(X, T) \backslash \Delta_{X}\right)>0$, As $X$ is a compact metric space there exists a closed set $F \subset \operatorname{supp}(\mu) \cap B P(X, T) \backslash \Delta_{X}$ with $\mu(F)>0$. By the ergodic decomposition theorem, there exists an ergodic measure $v$ with $v(F)>0$. By Birkhoff ergodic theorem there exists $z \in F$ such that

$$
\frac{1}{n} \#(N(z, F) \cap[0, n-1])=\frac{1}{n} \sum_{i=0}^{n-1} \chi_{F}\left(T^{i} z\right) \rightarrow \int \chi_{F} \mathrm{~d} v=v(F)>0,
$$

then we have $D(N(z, F))>0$. We choose neighborhoods $U$ and $V$ of $F$ and $\Delta_{X}$ respectively with $U \cap V=\emptyset$, then $\underline{B D}(N(z, U)) \geq D(N(z, F))>0$. On the other hand, we have $B D(N(z, V))=1$, since $z \in B P(X, T)$. The contradiction shows the lemma. 
For a minimal system the following result was known, see [2] and [12]. We now show it holds for a general system.

Theorem 4.3. Let $(X, T)$ be a dynamical system. Let $(Z, S)$ be the maximal equicontinuous factor of $(X, T)$ and $\pi:(X, T) \rightarrow(Z, S)$ be the factor map. Then the following conditions are equivalent:

(1) $(X, T)$ is mean equicontinuous;

(2) $\pi$ is Banach proximal;

(3) $B P(X, T)=P(X, T)=Q(X, T)$;

(4) $\pi:(X, \mu, T) \rightarrow(Z, v, S)$ is measure-theoretically isomorphic, where $\mu$ and $v$ are any invariant measures on $X$ and $Z$ respectively with $\pi(\mu)=v$;

(5) $(X, T)$ is equicontinuous in the mean.

Proof. (1) $\Rightarrow$ (2) by Lemma 3.4. (1) $\Leftrightarrow$ (5) by Theorem 3.8. Moreover, it is clear that (2) $\Leftrightarrow(3)$.

$(3) \Rightarrow(4)$ This is essentially proved in [12, Theorem 3.8]. Here we provide a proof for completeness.

Let $\mu$ be an invariant measure on $(X, T)$ and $v$ be the invariant measure on $(Z, S)$ with $\pi(\mu)=v$. We consider the disintegration of $\mu$ over $v$. That is, for a.e. $y \in Z$ we have a measure $\mu_{y}$ on $X$ such that $\operatorname{supp}\left(\mu_{y}\right) \subset \pi^{-1}(y)$ and $\mu=\int_{y \in Z} \mu_{y} \mathrm{~d} v$. Let $W=\{(u, v) \in X \times$ $X: \pi(u)=\pi(v)\}$. As $\operatorname{supp}\left(\mu_{y}\right) \subset \pi^{-1}(y)$, we have $\operatorname{supp}\left(\mu_{y} \times \mu_{y}\right) \subset \pi^{-1}(y) \times \pi^{-1}(y) \subset W$, a.e. $y \in Y$. Let $\mu \times_{Z} \mu=\int_{y \in Z} \mu_{y} \times \mu_{y} \mathrm{~d} v$. Then $\mu \times_{Z} \mu$ is an invariant measure on $(X \times X, T \times T)$. Moreover,

$$
\mu \times_{Z} \mu(W)=\int_{y \in Z} \mu_{y} \times \mu_{y}(W) \mathrm{d} v=1
$$

then $\operatorname{supp}\left(\mu \times_{Z} \mu\right) \subset W$. By Lemma 4.2 we have $\mu \times_{Z} \mu\left(\Delta_{X}\right)=1$. Since

$$
\mu \times_{Z} \mu\left(\Delta_{X}\right)=\int_{y \in Y} \mu_{y} \times \mu_{y}\left(\Delta_{X}\right) \mathrm{d} v=1,
$$

we have $\mu_{y} \times \mu_{y}\left(\Delta_{X}\right)=1$ a.e. $y \in Z$. Then for a.e. $y \in Z$, there exists a point $c_{y} \in \pi^{-1}(y)$ such that $\mu_{y}=\delta_{c_{y}}$.

Let $Y_{0}$ be the collection of $y \in Z$ such that $\mu_{y}$ is not equal to $\delta_{x}$ for any $x \in X$. Then $v\left(\cup_{i \in \mathbb{Z}_{+}} S^{-i} Y_{0}\right)=0$. Let $Z_{0}=Z \backslash \cup_{i \in \mathbb{Z}_{+}} S^{-i} Y_{0}$ and $X_{0}=\left\{c_{y}: y \in Z_{0}\right\}$. Then $v\left(Z_{0}\right)=1$. Now we show that $X_{0}$ is a measurable set. In fact, the map $y \mapsto \mu_{y}$ from $Z_{0}$ to $M(X)$ is measurable and $x \mapsto \delta_{x}$ is an embedding. Since $Z_{0}$ is a measurable set and maps are $1-1$, it follows from Souslin's theorem that $X_{0}$ is a measurable set, and it is clear that $\mu\left(X_{0}\right)=\mu\left(\pi^{-1} Z_{0}\right)=v\left(Z_{0}\right)=1$. By the same argument, $\pi:\left(X_{0}, \mu, T\right) \rightarrow\left(Z_{0}, v, S\right)$ is a measure-theoretic isomorphism.

(4) $\Rightarrow(5)$ If $(X, T)$ is not equicontinuous in the mean, then there are $x_{k}, x \in X, n_{k} \in$ $\mathbb{Z}_{+}, k=1,2, \cdots$ and $\varepsilon_{0}>0$ such that $\lim _{k \rightarrow \infty} x_{k}=x$ and for every $k \in \mathbb{Z}_{+}$

$$
\frac{1}{n_{k}} \sum_{i=0}^{n_{k}-1} d\left(T^{i} x_{k}, T^{i} x\right) \geq \varepsilon_{0} .
$$

Let $\pi\left(x_{k}\right)=z_{k}$ and $\pi(x)=z$. We define

$$
\mu_{k}=\frac{1}{n_{k}} \sum_{i=0}^{n_{k}-1} \delta_{\left(T^{i} x_{k}, T^{i} x\right)}
$$


and

$$
v_{k}=\frac{1}{n_{k}} \sum_{i=0}^{n_{k}-1} \delta_{\left(S_{z_{k}}, S_{z} i\right)}
$$

then

$$
(\pi \times \pi)\left(\mu_{k}\right)=v_{k}
$$

By taking the subsequence, there exists $\mu$ and $v$ on $M(X \times X, T \times T)$ and $M(Z \times Z, S \times S)$ respectively with $\lim _{k \rightarrow \infty} \mu_{k}=\mu, \lim _{k \rightarrow \infty} v_{k}=v$ and $(\pi \times \pi)(\mu)=v$.

We claim that $\mu\left(\operatorname{supp}(\mu) \backslash \Delta_{X}\right)>0$. Actually, $d(\cdot, \cdot)$ is a continuous function on $X \times X$, then we have

$$
\int_{X \times X} d(x, y) \mathrm{d} \mu_{k} \rightarrow \int_{X \times X} d(x, y) \mathrm{d} \mu
$$

and $\int_{X \times X} d(x, y) \mathrm{d} \mu_{k}=\frac{1}{n_{k}} \sum_{i=0}^{n_{k}-1} d\left(T^{i} x_{k}, T^{i} x\right) \geq \varepsilon_{0}$ which implies $\mu\left(\operatorname{supp}(\mu) \backslash \Delta_{X}\right)>0$. There are open sets $U$ and $V$ of $X$ with $U \cap V=\emptyset$ and $\mu(U \times V)>0$.

Let $\mu^{\prime}$ and $\nu^{\prime}$ be the projection of $\mu$ and $v$ onto the first component of $X$ and $Z$ respectively. It is clear that $\mu^{\prime} \in M(X, T)$ and $v^{\prime} \in M(Z, S)$. It is easy to see $\operatorname{supp}(v) \subset$ $\overline{\operatorname{Orb}((z, z), S \times S)}$. Then $v^{\prime}(\overline{\operatorname{Orb}(z, S)})=v(\overline{\operatorname{Orb}(z, S)} \times Z)=1$, which implies $\operatorname{supp}\left(v^{\prime}\right) \subset$ $\overline{\operatorname{Orb}(z, S)}$. Furthermore, $v^{\prime}$ is the only invariant measure on $\overline{\operatorname{Orb}(z, S)}$, since $\overline{\operatorname{Orb}(z, S)}$ is uniquely ergodic. As for every $f, g \in C(Z)$, we have

$$
\int_{Z \times Z} f\left(z_{1}\right) g\left(z_{2}\right) \mathrm{d} v_{k}\left(z_{1}, z_{2}\right) \longrightarrow \int_{Z \times Z} f\left(z_{1}\right) g\left(z_{2}\right) \mathrm{d} v\left(z_{1}, z_{2}\right)
$$

and

$$
\int_{Z \times Z} f\left(z_{1}\right) g\left(z_{2}\right) \mathrm{d} v_{k}\left(z_{1}, z_{2}\right)=\frac{1}{n_{k}} \sum_{i=0}^{n_{k}-1} f\left(S^{i} z_{k}\right) g\left(S^{i} z\right) \longrightarrow \int_{Z} f(z) g(z) \mathrm{d} v^{\prime}(z),
$$

thus $v(A \times B)=v^{\prime}(A \cap B)$. So $v^{\prime}(\pi(U) \cap \pi(V))=v(\pi(U) \times \pi(V)) \geq \mu(U \times V)>0$.

Obviously, $\pi\left(\mu^{\prime}\right)$ is an invariant measure on $\overline{\operatorname{Orb}(z, S)}$, thus we have $\pi\left(\mu^{\prime}\right)=v^{\prime}$. As $\pi:\left(X, \mu^{\prime}, T\right) \rightarrow\left(Z, v^{\prime}, S\right)$ is measure-theoretic isomorphic, we have $v^{\prime}(\pi(U) \cap \pi(V))=$ $\mu^{\prime}(U \cap V)=0$, it is a contradiction. This shows $(X, T)$ is mean equicontinuous.

\section{MEAN EQUiCONTINUITY AND WEYL MEAN EQUICONTINUITY}

Following [2] and [12], a dynamical system $(X, T)$ is called Banach mean equicontinuous or Weyl mean equicontinuous if for every $\varepsilon>0$, there exists a $\delta>0$ such that

$$
\limsup _{n-m \rightarrow \infty} \frac{1}{n-m} \sum_{i=m}^{n-1} d\left(T^{i} x, T^{i} y\right)<\varepsilon
$$

for all $x, y \in X$ with $d(x, y)<\delta$. It is clear that each Weyl mean equicontinuous system is mean equicontinuous. It is shown in [2] that if a minimal system is mean equicontinuous then it is Weyl mean equicontinuous. In this section we show that for a general dynamical system mean equicontinuity is equivalent to Weyl mean equicontinuity. That is, we have

Theorem 5.1. A dynamical system $(X, T)$ is mean equicontinuous if and only if it is Weyl mean equicontinuous.

Before proving the Theorem, we need the following lemma. 
Lemma 5.2. If a dynamical system $(X, T)$ is uniquely ergodic, then for any $f \in C(X, \mathbb{R})$ and $x \in X$,

$$
\lim _{n-m \rightarrow \infty} \frac{1}{n-m} \sum_{i=m}^{n-1} f\left(T^{i} x\right)=\lim _{n \rightarrow \infty} \frac{1}{n} \sum_{i=0}^{n-1} f\left(T^{i} x\right) .
$$

Proof. Let $\mu$ be the unique invariant measure on $(X, T)$. Then for any $f \in C(X, \mathbb{R})$ and $x \in X$,

$$
\lim _{n \rightarrow \infty} \frac{1}{n} \sum_{i=0}^{n-1} f\left(T^{i} x\right)=\int f \mathrm{~d} \mu .
$$

If the conclusion does not hold, then there exist $f \in C(X, \mathbb{R}), x \in X$ and two sequences $\left\{n_{k}\right\}$ and $\left\{m_{k}\right\}$ with $n_{k}-m_{k} \rightarrow \infty$ such that

$$
\lim _{n_{k}-m_{k} \rightarrow \infty} \frac{1}{n_{k}-m_{k}} \sum_{i=m_{k}}^{n_{k}-1} f\left(T^{i} x\right)=c \neq \int f \mathrm{~d} \mu .
$$

As $M(X)$ is compact, passing to a subsequence if necessary we may assume that

$$
\lim _{k \rightarrow \infty} \frac{1}{n_{k}-m_{k}} \sum_{i=m_{k}}^{n_{k}-1} \delta_{T^{i} x}=v .
$$

It is easy to see that $v$ is an invariant measure. As $(X, T)$ is unqiuely ergodic then $v=\mu$. So

$$
\lim _{n_{k}-m_{k} \rightarrow \infty} \frac{1}{n_{k}-m_{k}} \sum_{i=m_{k}}^{n_{k}-1} f\left(T^{i} x\right)=\int f \mathrm{~d} \mu .
$$

This is a contradiction.

Proof of Theorem 5.1. As $(X, T)$ is mean equicontinuous, then so is $(X \times X, T \times T)$. Fix $(x, y) \in X \times X$. By Theorem 3.6, $(\overline{\operatorname{Orb}((x, y), T \times, T)}, T \times T)$ is uniquely ergodic. Now applying the above theorem to the distance function $d(\cdot, \cdot)$ and $(x, y)$, we get

$$
\lim _{n-m \rightarrow \infty} \frac{1}{n-m} \sum_{i=m}^{n-1} d\left(T^{i} x, T^{i} y\right)=\lim _{n \rightarrow \infty} \frac{1}{n} \sum_{i=0}^{n-1} d\left(T^{i} x, T^{i} y\right) .
$$

Then the result follows from the definition.

We now give the following conclusion to end this section.

Theorem 5.3. Let $(X, T)$ be a mean equicontinuous system, then for every $\varepsilon>0$, there are $\delta>0$ and $N>0$, such that whenever $d(x, y)<\delta$, we have

$$
\frac{1}{n} \sum_{i=j}^{n+j-1} d\left(T^{i} x, T^{i} y\right)<\varepsilon
$$

for all $n \geq N$ and $j \geq 0$.

Proof. Assume that there are $x_{k}, y_{k}, z \in X, n_{k}, j_{k} \in \mathbb{Z}_{+}, k=1,2, \cdots$ and $\varepsilon_{0}>0$ such that $\lim _{k \rightarrow \infty} x_{k}=z=\lim _{k \rightarrow \infty} y_{k}$ and for every $k \in \mathbb{Z}_{+}$

$$
\frac{1}{n_{k}} \sum_{i=j_{k}}^{n_{k}+j_{k}-1} d\left(T^{i} x_{k}, T^{i} y_{k}\right) \geq \varepsilon_{0} \text {. }
$$


Let $\mu_{k}=\frac{1}{n_{k}} \sum_{i=j_{k}}^{n_{k}+j_{k}-1} \delta_{\left(T^{i} x_{k}, T^{i} y_{k}\right)}$, and then $\mu_{k} \in M(X \times X)$. We may assume $\mu_{k} \rightarrow$ $\mu$ (otherwise we may consider the subsequence), where $\mu \in M(X \times X, T \times T)$.

We claim that $\mu\left(\operatorname{supp}(\mu) \backslash \Delta_{X}\right)>0$. In fact, $d(\cdot, \cdot)$ is a continuous function on $X \times X$, then we have

$$
\int_{X \times X} d(x, y) \mathrm{d} \mu_{k} \longrightarrow \int_{X \times X} d(x, y) \mathrm{d} \mu
$$

and

$$
\int_{X \times X} d(x, y) \mathrm{d} \mu_{k}=\frac{1}{n_{k}} \sum_{i=j_{k}}^{n_{k}+j_{k}-1} d\left(T^{i} x_{k}, T^{i} y_{k}\right) \geq \varepsilon_{0}
$$

which implies

$$
\mu\left(\operatorname{supp}(\mu) \backslash \Delta_{X}\right)>0 .
$$

By ergodic decomposition theorem, we have $v\left(\operatorname{supp}(\mu) \backslash \Delta_{X}\right)>0$ for some ergodic measure $v$ on $X \times X$, thus there exists a minimal point $(u, v)$ in $\operatorname{supp}(\mu) \backslash \Delta_{X}$ since $\operatorname{supp}(v)$ is a minimal set by Lemma 3.7.

Let $B_{l}=\left\{(x, y) \in X \times X: d((x, y),(u, v))<\frac{1}{l}\right\}$. Then we have

$$
\mu\left(B_{l}\right)>0 \text { and } \mu_{k}\left(B_{l}\right)=\frac{1}{n_{k}} \#\left(\left\{j_{k} \leq i \leq n_{k}+j_{k}-1:\left(T^{i} x_{k}, T^{i} y_{k}\right) \in B_{l}\right\}\right) .
$$

Thus for any $l \in \mathbb{Z}_{+}$there exist infinte $k \in \mathbb{Z}_{+}$with $j_{k} \leq m_{k} \leq n_{k}+j_{k}-1$ such that $\left(T^{m_{k}} x_{k}, T^{m_{k}} y_{k}\right) \in B_{l}$, since $0<\mu\left(B_{l}\right) \leq \liminf _{k \rightarrow \infty} \mu_{k}\left(B_{l}\right)$.

Put

$$
\delta=d(\overline{\operatorname{Orb}((z, z), T \times T)}, \overline{\operatorname{Orb}((u, v), T \times T)})
$$

then $\delta>0$, since $\overline{\operatorname{Orb}((u, v), T \times T)} \cap \Delta_{X}=\emptyset$. As $(X, T)$ is mean equicontinuous, so is $(X \times X, T \times T)$ by Lemma 3.1. Thus, for $\frac{1}{4} \delta$, there is $\eta>0$ such that if $d\left((x, y),\left(x^{\prime}, y^{\prime}\right)\right)<$ $\eta$ then

$$
\limsup _{n \rightarrow \infty} \frac{1}{n} \sum_{i=0}^{n-1} d\left((T \times T)^{i}(x, y),(T \times T)^{i}\left(x^{\prime}, y^{\prime}\right)\right)<\frac{\delta}{4} .
$$

There are infinitely many $k \in \mathbb{Z}_{+}$with $d\left(\left(x_{k}, y_{k}\right),(z, z)\right)<\eta$ and $d\left(\left(T^{m_{k}} x_{k}, T^{m_{k}} y_{k}\right),(u, v)\right)<$ $\eta$, then

$$
\limsup _{n \rightarrow \infty} \frac{1}{n} \sum_{i=0}^{n-1} d\left((T \times T)^{i}\left(x_{k}, y_{k}\right),(T \times T)^{i}(z, z)\right)<\frac{\delta}{4}
$$

and

$$
\limsup _{n \rightarrow \infty} \frac{1}{n} \sum_{i=0}^{n-1} d\left((T \times T)^{i}\left(T^{m_{k}} x_{k}, T^{m_{k}} y_{k}\right),(T \times T)^{i}(u, v)\right)<\frac{\delta}{4}
$$

which implies

$$
\limsup _{n \rightarrow \infty} \frac{1}{n} \sum_{i=0}^{n-1} d\left((T \times T)^{i}\left(T^{m_{k}} z, T^{m_{k}} z\right),(T \times T)^{i}(u, v)\right)<\frac{\delta}{2} .
$$

It is a contradiction which shows the theorem. 


\section{Mean equicontinuous Relation}

It is well known that the equicontinuous structure relation is the smallest closed invariant equivalence relation containing the regionally proximal relation. In [12] the authors showed that each topological dynamical system admits a maximal mean equicontinuous factor. Inspired by the above ideas, we now define a new notation called a pair sensitive in the mean and introduce the mean equicontinuous structure relation. We show that the maximal mean equicontinuous factor is induced by the smallest invariant closed equivalence relation containing the relation of sensitivity in the mean.

Definition 6.1. Let $(X, T)$ be a dynamical system. We say $(x, y)$ is a pair sensitive in the mean, if $x=y$ or for any $\tau>0$, there exists $c=c(\tau)>0$ such that for every $\varepsilon>0$, there exist $x^{\prime}, y^{\prime} \in X$ and $n \in \mathbb{N}$ such that $d\left(x^{\prime}, y^{\prime}\right)<\varepsilon$ and

$$
\frac{1}{n} \#\left(\left\{0 \leq i \leq n-1: d\left(T^{i} x^{\prime}, x\right)<\tau, d\left(T^{i} y^{\prime}, y\right)<\tau\right\}\right)>c
$$

Let $Q_{m e}(X, T)$ be the set of all pairs sensitive in the mean in $(X, T)$, and we call that the relation of sensitivity in the mean.

Clearly, if $T$ is a homeomorphism, then $Q_{m e}(X, T) \subset Q(X, T)$. Let $S_{m e}(X, T)$ be the smallest closed $T \times T$ invariant equivalence relation such that $X / S_{m e}(X, T)$ is a mean equicontinuous system. We will show that $S_{m e}(X, T)$ is the smallest closed $T \times T$ invariant equivalent relation that contains the relation of sensitivity in the mean. This will be done through the following lemmas.

First we observe that

Lemma 6.2. Let $(X, T)$ be a dynamical system. Then $(X, T)$ is not mean equicontinuous system if and only if there exists $x, x_{k} \in X, n_{k} \in \mathbb{N}$ and $\varepsilon_{0}>0$, such that $x_{k} \rightarrow$ $x, \frac{1}{n_{k}} \sum_{i=0}^{n_{k}-1} d\left(T^{i} x, T^{i} x_{k}\right) \geq \varepsilon_{0}$.

It is easy to check:

Lemma 6.3. Let $\pi:(X, T) \rightarrow(Y, S)$ be a factor map. If $(x, y) \in Q_{m e}(X, T)$, then we have $(\pi(x), \pi(y)) \in Q_{m e}(Y, S)$.

Lemma 6.4. Let $(X, T)$ be a dynamical system, then $(X, T)$ is mean equicontinuous if and only if $Q_{m e}(X, T)=\Delta_{X}$.

Proof. If $(X, T)$ is mean equicontinuous, it is clear that $Q_{m e}(X, T)=\Delta_{X}$.

Conversely, assume that $Q_{m e}(X, T)=\Delta_{X}$. Suppose that $(X, T)$ is not mean equicontinuous. By Lemma 6.2, there are $x_{k}, x \in X, n_{k} \in \mathbb{Z}_{+}$and $\varepsilon_{0}>0$ such that $\lim _{k \rightarrow \infty} d\left(x_{k}, x\right)=0$ and for every $k \in \mathbb{N}$, we have

$$
\frac{1}{n_{k}} \sum_{i=0}^{n_{k}-1} d\left(T^{i} x_{k}, T^{i} x\right) \geq \varepsilon_{0} .
$$

Let $\mu_{k}=\frac{1}{n_{k}} \sum_{i=0}^{n_{k}-1} \delta_{\left(T^{i} x_{k}, T^{i} x\right)}$, then $\mu_{k} \in M(X \times X)$, we may assume $\mu_{k} \rightarrow \mu$ (otherwise we may consider the subsequence), where $\mu \in M(X \times X, T \times T)$. From the proof of Theorem 3.8, it follows that $\mu\left(\operatorname{supp}(\mu) \backslash \Delta_{X}\right)>0$. 
Let $(y, z) \in \operatorname{supp}(\mu) \backslash \Delta_{X}$. Fix $\tau>0$, choose $l \in \mathbb{N}$ such that $\frac{1}{l}<\tau$. Let $B_{l}=\{(u, v) \in$ $\left.X \times X: d((y, z),(u, v))<\frac{1}{l}\right\}$, where $d((y, z),(u, v))=d(y, u)+d(z, v)$ then

$$
\mu\left(B_{l}\right)>0 \text { and } \mu_{k}\left(B_{l}\right)=\frac{1}{n_{k}} \#\left(\left\{0 \leq i \leq n_{k}-1: d\left(T^{i} x_{k}, y\right)+d\left(T^{i} x, z\right)<\frac{1}{l}\right\}\right) .
$$

There exist infinite $k \in \mathbb{Z}_{+}$such that $\mu_{k}\left(B_{l}\right)>\frac{\mu\left(B_{l}\right)}{2}>0$ since $0<\mu\left(B_{l}\right) \leq \liminf _{k \rightarrow \infty} \mu_{k}\left(B_{l}\right)$. For $\varepsilon>0$, we can choose $k \in \mathbb{Z}_{+}$satisfying above proposition with $d\left(x_{k}, x\right)<\varepsilon$, hence

$$
\frac{1}{n_{k}} \#\left(\left\{0 \leq i \leq n_{k}-1: d\left(T^{i} x_{k}, y\right)<\tau, d\left(T^{i} x, z\right)<\tau\right\}\right)>\frac{\mu\left(B_{l}\right)}{2} .
$$

It follows that $(y, z) \in Q_{m e}(X, T)$. It is a contradiction which implies the lemma.

Lemma 6.5. Let $(X, T)$ be a dynamical system and $\mathscr{A}\left(Q_{m e}(X, T)\right)$ be the smallest closed $T \times T$ invariant equivalence relation containing $Q_{m e}(X, T)$, then $X / \mathscr{A}\left(Q_{m e}(X, T)\right)$ is the maximal mean equicontinuous factor.

Proof. Let $Y=X / \mathscr{A}\left(Q_{m e}(X, T)\right)$ and $\pi: X \rightarrow Y$ be the factor map. As $\pi: X \rightarrow Y$ is a continuous surjective, we can choose a metric on $X$ (also denoted by $d$ ) such that $d(x, y) \geq$ $d(\pi(x), \pi(y))$ for all $x, y \in X$.

Assume that $(Y, T)$ is not mean equicontinuous. By Lemma 6.4, there exist $x, y \in Y$ with $x \neq y$ and $(x, y) \in Q_{m e}(Y, T)$. Let $\tau<\frac{1}{4} d(x, y)$. For $k \in \mathbb{N}$, there are $x_{k}, y_{k} \in Y$ and $n_{k} \in \mathbb{Z}_{+}$with $d\left(x_{k}, y_{k}\right)<\frac{1}{k}$ such that

$$
\frac{1}{n_{k}} \#\left(\left\{0 \leq i \leq n_{k}-1: d\left(T^{i} x_{k}, x\right)<\tau, d\left(T^{i} y_{k}, y\right)<\tau\right\}\right)>c
$$

for some $c>0$. For every $k \in \mathbb{N}$, choose $u_{k}, v_{k} \in X$ such that $\pi\left(u_{k}\right)=x_{k}, \pi\left(v_{k}\right)=y_{k}$. Without loss of generality, we can assume that $\lim _{k \rightarrow \infty} x_{k}=z=\lim _{k \rightarrow \infty} y_{k}$ and $\lim _{k \rightarrow \infty} u_{k}=w=$ $\lim _{k \rightarrow \infty} v_{k}$, then $\pi(w)=z$.

$$
\frac{1}{n_{k}} \sum_{i=0}^{n_{k}-1} d\left(T^{i} u_{k}, T^{i} v_{k}\right) \geq \frac{1}{n_{k}} \sum_{i=0}^{n_{k}-1} d\left(T^{i} x_{k}, T^{i} y_{k}\right) \geq c \cdot(d(x, y)-2 \tau)>\frac{c}{2} \cdot d(x, y)>0 .
$$

Let $\mu_{k}=\frac{1}{n_{k}} \sum_{i=0}^{n_{k}-1} \delta_{\left(T^{i} u_{k}, T^{i} v_{k}\right)}$ and $v_{k}=\frac{1}{n_{k}} \sum_{i=0}^{n_{k}-1} \delta_{\left(T^{i} x_{k}, T^{i} y_{k}\right)}$. Without loss of generality, assume that $\mu_{k} \rightarrow \mu$ and $v_{k} \rightarrow v$, where $\mu \in M(X \times X, T \times T)$ and $v \in M(Y \times Y, T \times T)$. From the proof of Theorem 3.8, we have $\mu\left(\operatorname{supp}(\mu) \backslash \Delta_{X}\right)>0$ and $v\left(\operatorname{supp}(v) \backslash \Delta_{Y}\right)>0$. Moreover $\pi \times \pi(\mu)=v$ since $\pi \times \pi\left(\mu_{k}\right)=v_{k}$.

If $(a, b) \in \operatorname{supp}(\mu)$, by the proof of Theorem 3.8, we have $(a, b) \in Q_{m e}(X, T)$ which implies $\pi(a)=\pi(b)$, hence $\pi \times \pi(\operatorname{supp}(\mu))=\Delta_{Y}$.

Therefore $v\left(\Delta_{Y}\right)=\pi \times \pi(\mu)\left(\Delta_{Y}\right)=\mu\left((\pi \times \pi)^{-1} \Delta_{Y}\right)=\mu(\operatorname{supp}(\mu))=1$.

It is a contradiction which implies the lemma.

By Lemma 6.4 and Lemma 6.5, we have the main result in this section.

Theorem 6.6. Let $(X, T)$ be a dynamical system. Then $S_{m e}(X, T)$ is the smallest closed $T \times T$ invariant equivalence relation containing $Q_{m e}(X, T)$.

Acknowledgments. The authors would like to thank Jian Li and Xiangdong Ye for bringing us the questions and for useful discussions when doing the research. We also thank Jie 
Li for the careful reading which help the writing of the paper. Finally the authors thank the referee for his/her careful reading.

The authors were supported by NNSF of China (11431012).

\section{REFERENCES}

1. J. Auslander, Mean-L-stable systems, Illinois J. Math. 3 (1959), 566-579.

2. T. Downarowicz and E. Glasner, Isomorphic extensions and applications, Topol, Methods Nonlinear Anal. 48 (2016), 321-338.

3. R. Ellis and W. H. Gottschalk, Homomorphisms of transformation groups, Trans. Amer. Math. Soc. 94 (1960), 258-271.

4. S. Fomin, On dynamical systems with a purely point spectrum, Dokl. Akad. Nauk SSSR, vol. 77 (1951), 29-32 (In Russian).

5. H. Furstenberg, Recurrence in Ergodic Theory and Combinatorial Number Theory, Princeton Univ. Press, Princeton, NJ, 1981.

6. F. Garcia-Ramos, A characterization of $\mu$-equicontinuity for topological dynamical systems, Proc. Amer. Math. Soc. 145 (2017), no. 8, 3357-3368.

7. F. Garcia-Ramos, Weak forms of topological and measure-theoretical equicontinuity: relationships with discrete spectrum and sequence entropy, Ergodic Theory Dynam. Systems 37 (2017), no. 4, 12111237.

8. F. Garcia-Ramos, L. Jin, Mean proximality and mean Li-Yorke chaos. Proc. Amer. Math. Soc, 145 (2017), no. 7, 2959-2969.

9. F. García-Ramos, J. Li, R. Zhang, When is a dynamical system mean sensitive?, Ergodic Theory Dynam. Systems, to appear (on line in 2017) .

10. W. Huang, J. Li, J. Thouvenot, L. Xu and X. Ye, Mean equicontinuity, bounded complexity and discrete spectrum, arXiv:1806.02980.

11. Jian Li and S. Tu, On proximality with Banach density one, J. Math.Anal.Appl. 416 (2014), 36-51.

12. Jian Li, S. Tu and X. Ye, Mean equicontinuity and mean sensitivity, Ergod. Th. Dynam. Sys. 35 (2015), 2587-2612.

13. J. Li, How chaotic is an almost mean equicontinuous system? DCDS-A., 38 (2018), no. 9, 4727-4744.

14. J. C. Oxtoby, Ergodic sets, Bull. Amer. Math. Soc., 58 (1952), 116-136.

15. B. Scarpellini, Stability properties of flows with pure point spectrum, J. London Math. Soc. (2) 26 (1982), no. 3, 451-464.

16. P. Walters, An introduction to ergodic theory. Graduate Texts in Mathematics, 79. Springer-Verlag, New York-Berlin, 1982.

17. X. Ye and R. Zhang, On sensitivity sets in topological dynamics, Nonlinearity, 21 (2008), 1601-1620.

(J. Qiu) Wu Wen-Tsun Key Laboratory of Mathematics, USTC, Chinese ACAdemy of Sciences and School of Mathematics, University of Science and Technology of China, HEFEI, ANHUi, 230026, P.R. CHINA

E-mail address: qiujh@mail.ustc.edu.cn

(J. Zhao) Wu Wen-Tsun Key Laboratory of Mathematics, USTC, Chinese Academy of SCIENCES AND SChool of Mathematics, University of SCIENCE AND TEChNOlogy of ChinA, HeFEI, ANHui, 230026, P.R. CHINA

E-mail address: zjianjie@mail.ustc.edu.cn 